International Mathematical Forum, 2, 2007, no. 21, 1021 - 1024

\title{
$k$-Perfect Geodomination is Affected by Adding a Pendant Vertex
}

\author{
Doost Ali Mojdeh and Nader Jafari Rad \\ Department of Mathematics \\ University of Mazandaran \\ Babolsar, P.O. Box 47416-1467, Iran
}

\begin{abstract}
A $k$-geodominating set is a geodominating set $S$ such that any vertex $v \in V(G) \backslash S$ is geodominated by a pair $x, y$ of vertices of $S$ with $d(x, y)=k$. A k-perfect geodominating set is a geodominating set $S$ such that any vertex $v \in V(G) \backslash S$ is geodominated by exactly one pair $x, y$ of the vertices of $S$ with $d(x, y)=k$. The cardinality of a minimum perfect geodominating set in $G$ is its perfect geodomination number $g_{p}(G)$ and the cardinality of a minimum k-perfect geodominating set in $G$ is its k-perfect geodomination number $g_{k p}(G)$. We investigate the affection of k-perfect geodomination numbers of a graph $G$ when a pendant vertex is added.
\end{abstract}

Mathematics Subject Classification: 05C69

Keywords: geodomination, k-geodomination, pendant vertex

\section{Introduction}

Let $G$ be a non-trivial connected graph. A $x-y$ path of length $d(x, y)$ is called a $x-y$ geodesic. A vertex $v$ is said to be in a $x-y$ geodesic $P$ if $v$ is an internal vertex of $P$. The closed interval $I[x, y]$ consists of $x, y$ and all vertices lying in some $x-y$ geodesic of $G$, while for $S \subseteq V(G)$,

$$
I[S]=\cup_{x, y \in S} I[x, y]
$$

A set $S$ of vertices is a geodetic set if $I[S]=V(G)$, and the minimum cardinality of a geodetic set is the geodetic number $g(G)$. A geodetic set of cardinality $g(G)$ is called a $g$-set, (see $[1,2,3,4,5,6])$. 
Geodetic concepts were studied from the point of view of domination. Geodetic sets and the geodetic number were referred to as geodominating sets and geodomination number [2] that we adopt in this paper.

A pair $x, y$ of vertices in a nontrivial connected graph $G$ is said to geodominate a vertex $v$ of $G$ if either $v \in\{x, y\}$ or $v$ lies in an $x-y$ geodesic of $G$. A set $S$ of vertices of $G$ is a geodominating set if every vertex of $G$ is geodominated by some pair of vertices of $S$. A vertex of $G$ is link-complete if the subgraph induced by its neighborhood is complete. It is easily seen that any link-complete vertex belongs to any geodominating set. For a graph $G$ and an integer $k \geq 1$, a vertex $v$ of $G$ is $k$-geodominated by a pair $x, y$ of distinct vertices in $G$ if $v$ is geodominated by $x, y$ and $d(x, y)=k$. A set $S$ of vertices of $G$ is a $k$-geodominating set of $G$ if each vertex $v$ in $V(G) \backslash S$ is $k$-geodominated by some pair of distinct vertices of $S$. The minimum cardinality of a $k$-geodominating set of $G$ is its $k$-geodomination number $g_{k}(G)$. A $k$-geodomination set of cardinality $g_{k}(G)$ is called a $g_{k}$-set of $G$. All graphs are connected and for an edge $e=\{u, v\}$ of a graph $G$ with $\operatorname{deg}(u)=1$ and $\operatorname{deg}(v)>1$, we call $e$ a pendant edge and $u$ a pendant vertex.

\section{How k-perfect geodomination is affected by adding a pendant vertex}

How $k$-geodomination numbers are affected by adding a vertex, was studied in [5]. Here we study this concept for $k$-perfect geodominating sets.

A perfect geodominating set is a geodominating set $S$ such that any vertex $v \in V(G) \backslash S$ is geodominated by exactly one pair $x, y$ of the vertices of $S$

Definition 2.1 Let $k \geq 1$ be an integer. A $k$-perfect geodominating; set is a geodominating set $S$ such that any vertex $v \in V(G) \backslash S$ is geodominated by exactly one pair $x, y$ of vertices of $S$ with $d(x, y)=k$.

The cardinality of a minimum $k$-perfect geodominating set in $G$ is its $k$-perfect geodomination number $g_{k p}(G)$.

Theorem 2.2 Let $G$ be a graph with no pendant vertices and $G^{\prime}$ obtained from $G$ by adding a pendant edge uv with $v \notin G, u \in G$, and also the vertex $u$ belongs to some $g_{p}(G)$-set. Then $g_{p}\left(G^{\prime}\right)=g_{p}(G)$.

Proof Let $S$ be a $g_{p}(G)$-set containing the vertex $u$, then it is easily seen that $(S \backslash\{u\}) \cup\{v\}$ is a perfect geodominating set for $G^{\prime}$. So $g_{p}\left(G^{\prime}\right) \leq g_{p}(G)$. Now if $S^{\prime}$ is a $g_{p}\left(G^{\prime}\right)$-set with size less than $g_{p}(G)$ then $v \in S^{\prime}$, so $\left(S^{\prime} \backslash\{v\}\right) \cup$ $\{u\}$ is a $g_{p}(G)$-set with size less than $g_{p}(G)$, which is a contradiction. Hence $g_{p}\left(G^{\prime}\right) \geq g_{p}(G)$ and the Proof is complete. 
Note that the condition $u$ belongs to some $g_{p}(G)$-set in the above proposition is necessary. For this order consider the following:

Example 2.3 Let $H$ be the graph $C_{2 n}$ with vertices $v_{1}, v_{2}, \ldots, v_{n}$. Add ears $v_{1}, w_{2}, w_{3}, \ldots, w_{n}, v_{n+1}$ and $v_{1}, w_{2}^{\prime}, w_{3}^{\prime}, . ., w_{n}, v_{n+1}$ to obtain a graph $G$ with $g_{p}(G)=$ 2. Now if $G^{\prime}$ obtained from $G$ by adding one of the pendant edges $x w_{i}, 2 \leq i \leq$ $n, x \notin V(G)$, then $g_{p}\left(G^{\prime}\right)>g_{p}(G)$.

To adding of a pendant edge to a connected graph $G$ can produce a graph $H$ with $g_{k p}(H)>g_{k p}(G)$.

Example 2.4 Consider the cycle $C_{6}: v_{1}, v_{2}, \ldots, v_{6}, v_{1}$ and add a pendant edge $v_{6} u$ with $u \notin C_{6}$, and also add $m \geq 4$ ears $v_{1}, w_{i 2}, w_{i 3}, v_{4}$ for $i=1,2, \ldots, m$ to obtain a graph $G$. Now let $G^{\prime}$ obtained from $G$ by adding the pendant edge $v_{4} w$ with $w \notin V(G)$, then $g_{3 p}\left(G^{\prime}\right)=2+g_{3 p}(G)$. So the addition of a pendant edge to a connected graph $G$ can strictly increase its $k$-perfect geodomination number.

Also the addition of a pendant edge to a connected graph $G$ can produce a graph whose $k$-perfect geodomination number is strictly smaller than that of G.

Example 2.5 Consider the cycle $C_{2 m}: v_{1}, v_{2}, \ldots, v_{2 m}, v_{1}$ for some $m \geq 2$. Since $\operatorname{diam}\left(C_{2 m}\right)=m$, then $g_{(m+1) p}\left(C_{2 m}\right)=2 m$. Let $G$ be a graph obtained from $C_{2 m}$ by adding the pendant edge $u v_{m+1}$. Since $\left\{u, v_{1}\right\}$ is a $(m+1)$-perfect geodominating set, then $g_{(m+1) p}(G)=2$.

Theorem 2.6 For every pair $k, n$ of positive integers there exists connected graphs $G$ and $G^{\prime}$ where $G^{\prime}$ is obtained from $G$ by adding a vertex $v$ with more than one edge incident with $v$ and $g_{k p}(G)-g_{k p}\left(G^{\prime}\right)=n$.

Proof Let $P_{i}: v_{i 1}, v_{i 2}, \ldots, v_{i(k-1)}$ be a path of order $k-1$ for $i=1,2, \ldots, n$ and let $G$ be obtained from the paths $P_{i}$ by adding a new vertex $v$ and joining $v$ to each of the vertices $v_{i 1}$ for $i=1,2, \ldots, n$. It is easily seen that $\left\{v_{i(k-1)}\right.$ : $i=1,2, \ldots, n\} \cup\left\{v_{11}, v_{21}\right\}$ is a $k$-perfect geodominating set of $G$. Moreover $g_{k p}(G)=n+2$. Now let $G^{\prime}$ obtained from $G$ by adding a new vertex $u$ and joining it to each of the vertices $v_{i(k-1)}$ for $i=1,2, \ldots, n$. Thus $d(u, v)=k$ and $g\left(G^{\prime}\right)=2$, so $g_{k p}\left(G^{\prime}\right)=2$. Hence $g_{k p}(G)-g_{k p}\left(G^{\prime}\right)=n$. 


\section{References}

[1] G.Chartrand, F.Harary and P.Zhang, Geodetic sets in graphs, Discussiones Mathematicae graph theory, 20 (2000), 129 - 138.

[2] G.Chartrand, F.Harary, H.C.Swart and P.Zhang, Geodomination in graphs, Bull.ICA, 31 (2001), 51 - 59.

[3] G.Chartrand and P.Zhang, The forcing geodetic number of a graph, Discussiones Mathematicae Graph theory, 19(1999), 45 - 58.

[4] D. A. Mojdeh and N. Jafari Rad, (k-)Perfect geodominating sets in graphs. to appear Oppuscula mathemaicae.

[5] R. Muntean and P.Zhang, k-geodomination in Graphs, ARS Combinatorica, 63 (2002), 33 - 47.

[6] R.Muntean and P.Zhang, On geodomination in graphs, Congressus Numerantium, 143 (2000), 161 - 174.

Received: August 15, 2006 